\title{
Contextual Pattern Matching ${ }^{\star}$
}

\author{
Gonzalo Navarro \\ $\mathrm{CeBiB}$ - Center for Biotechnology and Bioengineering, \\ Department of Computer Science, University of Chile. \\ Beauchef 851, Santiago, Chile. gnavarro@dcc.uchile.cl
}

\begin{abstract}
The research on indexing repetitive string collections has focused on the same search problems used for regular string collections, though they can make little sense in this scenario. For example, the basic pattern matching query "list all the positions where pattern $P$ appears" can produce huge outputs when $P$ appears in an area shared by many documents. All those occurrences are essentially the same.

In this paper we propose a new query that can be more appropriate in these collections, which we call contextual pattern matching. The basic query of this type gives, in addition to $P$, a context length $\ell$, and asks to report the occurrences of all distinct strings $X P Y$, with $|X|=|Y|=\ell$. While this query is easily solved in optimal time and linear space, we focus on using space related to the repetitiveness of the text collection and present the first solution of this kind. Letting $\bar{r}$ be the maximum of the number of runs in the BWT of the text $T[1 . . n]$ and of its reverse, our structure uses $O(\bar{r} \log (n / \bar{r}))$ space and finds the $c$ contextual occurrences $X P Y$ of $(P, \ell)$ in time $O(|P|+c \log n)$. We give other space/time tradeoffs as well, for compressed and uncompressed indexes.
\end{abstract}

\section{Introduction}

About a decade ago, it was realized that many of the fastest-growing text collections of the "data deluge" were highly repetitive [17. Since then, a number of research results have focused on developing indexes whose size is related to some good measure of compressibility for highly repetitive string collections [21]. Today one can find indexes built on measures like the size of the Lempel-Ziv parse [161194, of a grammar generating only the text 825], of a string attractor [237, the number of runs in the Burrows-Wheeler Transform (BWT) 6] of the text [1712, or the size of an automaton [5] recognizing text substrings [21].

All these indexes are devoted to the basic pattern matching query: given a short pattern string $P[1 . . m]$, output all the occ positions where it occurs in the text $T[1 . . n]$. Some indexes have managed to solve this problem in optimal time, $O(m+o c c)$, using space bounded by some function of the above measures [112], whereas others have low polylogarithmic factors multiplying $m$ or occ.

While very reasonable in general, this query can be pretty useless in a highly repetitive text collection. A pattern $P$ that appears inside a highly repeated

\footnotetext{
* Supported in part by Fondecyt grant 1-200038 and Basal Funds FB0001, Chile.
} 
text area will be reported myriad times, wasting a lot of effort to produce and to handle the result. We are not aware of many efforts to propose queries that are better adapted to a scenario of high repetitiveness.

In this paper we make a first step in this direction. We propose a query called contextual pattern matching which, in addition to $P$, gives a context length $\ell$. We then want one element of output per distinct context where $P$ appears, that is, all the positions where $P$ appears preceded by the same string $X$ of length $\ell$ and followed by the same string $Y$ of length $\ell$ shall be reported only once.

Definition 1. The contextual pattern matching problem on a text $T[1 . . n]$ is, given a pair $(P[1 . . m], \ell)$, return a position in $T$ for each of the $c$ distinct strings $X P Y$ occurring in $T$, for all $X, Y$ such that $|X|=|Y|=\ell$. For the occurrences near the extremes of $T$, assume $T$ is preceded and followed by $\ell$ copies of the special symbol $\$$, which cannot appear in $P$.

It is not hard to solve this query in optimal time $O(m+c)$ if we use linear space, $O(n)$, by using suffix trees 26 and other linear-space auxiliary structures. We are interested, however, in using space related to a relevant repetitiveness measure. We show that, if we call $\bar{r}$ the maximum of the number of equal-letter runs in the BWT of $T$ or its reverse, then a data structure using $O(\bar{r} \log (n / \bar{r}))$ space can solve contextual pattern matching in time $O(m+c \log n)$. We also show how any compressed text index can be extended with $O(n)$ bits and efficiently solve this query; this can be interesting for mildly repetitive texts.

\section{Preliminaries}

We index a text $T[0 . . n]$ over alphabet $[1 . . \sigma]$, where $T[0]=T[n]=\$$ is a special terminator smaller than all the other alphabet symbols. The suffix array [18] $S A[1 . . n]$ of $T$ lists all the suffixes $T[i . . n]$ for $i \geq 1$ in lexicographic order, and the $L C P$ array, $L C P[1 . . n]$, gives the length of the longest common prefix between consecutive suffix array entries, $L C P[i]=l c p(T[S A[i] . . n], T[S A[i-1] . . n])$.

One relevant measure of repetitiveness is called $r$, the number of equal-letter runs in the Burrows-Wheeler Transform (BWT) of $T[1 . . n]$. The BWT [6] is a reordering of the symbols of $T$ obtained by collecting the symbol preceding the lexicographically sorted suffixes of $T$. That is, if $S A[1 . . n]$ is the suffix array of $T$, then $B W T[i]=T[S A[i]-1]$. For example, it is known that $r=O\left(\gamma \log ^{2} n\right)$ [14, where $\gamma$ is the smallest attractor of $T[15]$.

Gagie et al. [12, Sec. 5.2-5.4 \& 6.3] describe data structures of size $O(r \log (n / r))$ that can find the suffix array range of any pattern $P[1 . . m]$ in time $O(m)$, and that can compute any entry $S A[i], S A^{-1}[i]$, and $L C P[i]$, in time $O(\log (n / r))$. Further, they can compute the following queries on $L C P, 1$

- RMQ $(i, j)=\operatorname{argmin}_{i \leq k \leq j} L C P[k]$ in time $O(\log (n / r))$.

- PSV $(p, d)=\max (\{q<p, L C P[q]<d\} \cup\{0\})$, in time $O\left(\log (n / r)+\log \log _{w} r\right)$.

- NSV $(p, d)=\min (\{q>p, L C P[q]<d\} \cup\{n+1\})$, in time $O(\log (n / r)+$ $\left.\log \log _{w} r\right)$.

\footnotetext{
${ }^{1}$ Our $P S V$ and $N S V$ are called $P S V^{\prime}$ and $N S V^{\prime}$ in there.
} 


\section{Our Solution}

We present a suffix-array-oriented solution that solves a stronger variant of the problem: we give the $c$ suffix array ranges of all the distinct contexts $X P Y$ where $P$ occurs in $T$. We can then report one text position for each, but also determine how many times each context occurs, and report its occurrences one by one.

We store the described data structures of Gagie et al. [12 for both $T[0 . . n]$ and its reverse $T^{r e v}[0 . . n]$. We call $r$ and $r^{\prime}$ the number of equal-letter runs in the BWT of $T$ and of $T^{r e v}$, respectively, and $\bar{r}=\max \left(r, r^{\prime}\right)$. Therefore the structures we use take space $O(\bar{r} \log (n / \bar{r}))$. The general strategy to solve a query $(P[1 . . m], \ell)$ is as follows:

1. We first find, in $O(m)$ time, the suffix array range [rs..re] of $P^{r e v}$ (i.e., $P$ read backwards) in the suffix array $S A^{\prime}$ of $T^{r e v}$.

2. We then partition $[r s . . r e]$ into $k \leq c$ maximal consecutive intervals $\left[r s_{i}, r e_{i}\right]$ where the suffixes in each interval share their first $m+\ell$ symbols, that is, $T^{r e v}\left[S A^{\prime}[p] . . S A^{\prime}[p]+m+\ell-1\right]=P^{r e v} X_{i}^{r e v}$ for all $r s_{i} \leq p \leq r e_{i}$.

3. We map each interval $S A^{\prime}\left[r s_{i}, r e_{i}\right]$ to the interval $S A\left[d s_{i} . . d e_{i}\right]$ corresponding to the suffixes that start with $X_{i} P$.

4. We partition each interval $S A\left[d s_{i} . . d e_{i}\right]$ into $k_{i}$ maximal consecutive subintervals $S A\left[d s_{i}^{j} . . d e_{i}^{j}\right]$ where the suffixes in each subinterval share their first $m+2 \ell$ symbols, $T[S A[p] . . S A[p]+m+2 \ell-1]=X_{i} P Y_{j}$ for all $d s_{i}^{j} \leq p \leq d e_{i}^{j}$.

5. We report the $c=\sum_{i=1}^{k} k_{i}$ resulting subintervals $S A\left[d s_{i}^{j} . . d e_{i}^{j}\right]$ and, if desired, a text position $S A[p]$ with $d s_{i}^{j} \leq p \leq d e_{i}^{j}$ for each.

We now solve the two nonobvious subproblems of our general strategy. The first, in points 2 and 4 , is to partition a suffix array interval into subintervals of suffixes sharing their first $t$ symbols. The second, in point 3, is how to map an interval of the suffix array of $T^{r e v}$ into the corresponding interval in the suffix array of $T$. The solutions we find have a complexity of $O(\log n)$ per item output, which leads to our promised result.

Theorem 1. Let $T$ be a text of length $n$, and let $\bar{r}$ be the maximum of the number of equal letter runs of its $B W T$ and the BWT of its reverse. Then there is a data structure of size $O(\bar{r} \log (n / \bar{r}))$ that finds the c contextual occurrences of $(P[1 . . m], \ell)$ in time $O(m+c \log n)$.

The data structures [12] can be built in $O(n)$ time and space, or in $O(n \log n)$ time and $O(\bar{r} \log (n / \bar{r}))$ space, the same as the final space of the structures.

Example. Figure 1 shows an example on the text $T[0 . .17]=\$$ alabaralalabarda $\$$, where we search for $P=$ a with context length $\ell=1$. Step 1 finds the interval $S A^{\prime}[$ rs..re $]=S A^{\prime}[2 . .9]$ of all the occurrences of $P^{r e v}=$ a on $T^{r e v}$. Step 2 finds the places where $L C P^{\prime}[p]<m+\ell=2$ (see Section 3.1), for $p \in[2 . .9]$, namely $2,3,5,6,9$. These are the starting positions of the intervals $\left[r s_{i}, r e_{i}\right]=$ $[2,2],[3,4],[5,5],[6,8],[9,9]$, and correspond to the contexts $P^{r e v} X_{i}^{r e v}=\mathrm{a} \$$, ab, ad, 


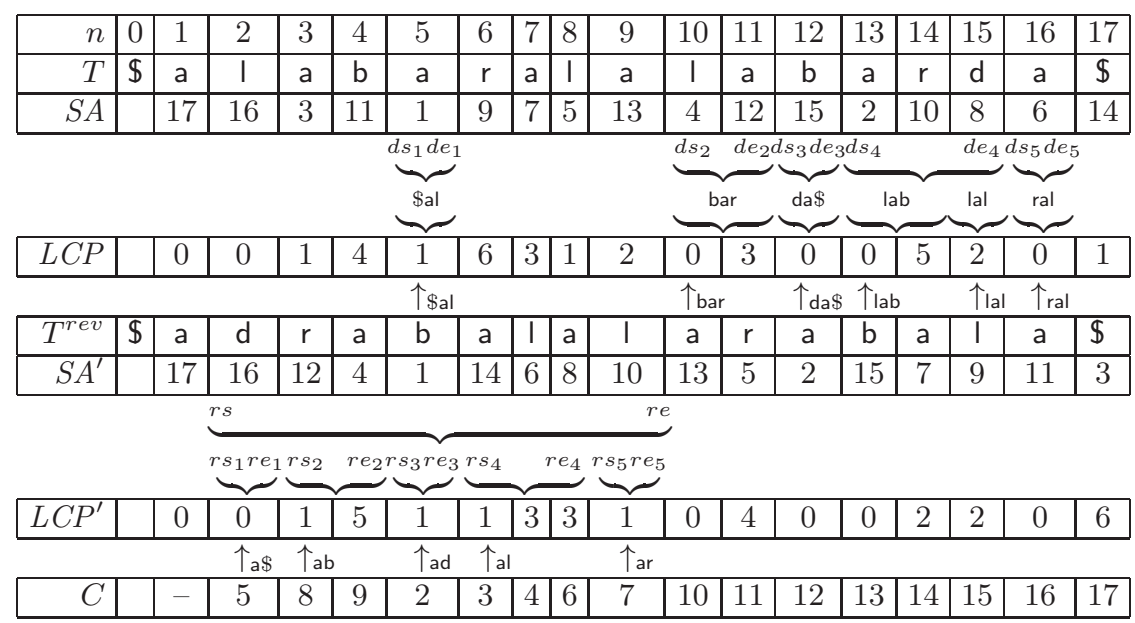

Fig. 1. Example trace.

al, ar. Step 3 maps those intervals to $S A$ (see Section 3.2), $\left[d s_{i}, d e_{i}\right]=[5,5],[10,11]$, $[12,12],[13,15],[16,16]$; they retain the same order of $S A^{\prime}$ only because $\ell=1$. Step 4 splits each interval at subintervals starting wherever $L C P[p]<m+2 \ell=3$, namely positions $5,10,12,13,15,16$. Therefore, the resulting subintervals (i.e., the output) are $[5,5],[10,11],[12,12],[13,14],[15,15],[16,16]$, corresponding to the contexts $\$ a l$, bar, da\$, lab, lal, ral.

We also show the array $C$ used in Section 3.3 note that each $d s_{i}$ corresponds to mapping the minimum position of $C$ in $\left[r s_{i}, r e_{i}\right]$.

\subsection{Partitioning a suffix array interval}

Given a range $[s . . e]$ of the suffix array of a string $S$, and a length $t$, we must partition it into maximal subranges $\left[s_{1} . . e_{1}\right], \ldots,\left[s_{k} . . e_{k}\right]$ where the suffixes starting in each subrange share their first $t$ symbols.

Note that $s_{1}=s$ and $s_{2}, \ldots, s_{k}$ are the values in $[s . . e]$ where $L C P[i]<t$, where $L C P$ is the LCP array of $S$. We use the standard procedure for 3 -sided queries to find all those positions $s_{i}$ : compute $p=R M Q(s, e)$ and, if $L C P[p]<t$, recurse on $[s, p-1]$, report $p$, and recurse on $[p+1, e]$. This process requires $O(k)$ computations of $R M Q$ and accesses to $L C P$ to find $s_{2}, \ldots, s_{k}$ in order, each of which takes time $O(\log (n / r))$, where $r$ is the number of runs in the BWT of $S$.

Since we use this technique for $S=T$ and $S=T^{r e v}$, the total cost is $O\left(k \max \left(\log (n / r), \log \left(n / r^{\prime}\right)\right)\right) \subseteq O(k \log n)$.

\subsection{Mapping suffix array intervals}

Given the suffix array interval $S A^{\prime}\left[s^{\prime} . . e^{\prime}\right]$ of $T^{r e v}$, consisting of all the suffixes that start with a string of length $t$, we want to find the corresponding suffix 
array interval $S A\left[\right.$ s..e] of $T$. With the suffix array $S A^{\prime}$ of $T^{r e v}$ and the inverse suffix array $S A^{-1}$ of $T$, we can translate any such suffix, say $p=S A^{-1}[n-$ $\left.S A^{\prime}\left[s^{\prime}\right]-(m+\ell-1)\right]$ (or $p=S A^{-1}[1]$ if $\left.n-S A^{\prime}\left[s^{\prime}\right]-(m+\ell-1) \leq 0\right)$. We indeed store the structures to compute those in time $O\left(\log (n / r)+\log \left(n / r^{\prime}\right)\right) \subseteq O(\log n)$ (Section 2).

We know that $s \leq p \leq e$, so the task is to extend $p$ in both directions: $s \leq p$ is the largest position where $L C P[s]<t$ and $e \geq p$ is the smallest position where $L C P[e+1]<t$. Those are, precisely, the operations $P S V(p, t)$ and $N S V(p, t)-1$ that our structures on $L C P$ compute in time $O\left(\log (n / r)+\log \log _{w} r\right) \subseteq O(\log n)$ (Section 2 again).

\subsection{Running on Other Indexes}

If we are willing to store uncompressed data structures of $O(n)$ space, we can find the interval of point (1) in RAM-optimal time $O\left(m / \log _{\sigma} n\right)$ using an enhanced suffix tree [22] on $T^{r e v}$. The $k$ intervals $\left[r s_{i}, r e_{i}\right]$ of point (2) can be found in $O(k)$ time using range minimum queries $R M Q^{\prime}(i, j)$ on the LCP array of $T^{r e v}, L C P^{\prime}$. Such queries take constant time using $2 n+o(n)$ bits of space [10. Each such interval $S A^{\prime}\left[r s_{i}, r e_{i}\right]$ can then be mapped (point 3) to $S A\left[d s_{i}, d e_{i}\right]$ by storing an array $C[1 . . n]$ with $C[i]=S A^{-1}\left[n-S A^{\prime}[i]\right]$ and building an $R M Q_{C}$ data structure on $C$, so that $d s_{i}=S A^{-1}\left[n-S A^{\prime}\left[R M Q_{C}\left(r s_{i}, r e_{i}\right)\right]-(m+\ell-1)\right]$ and $d e_{i}=d s_{i}+\left(r e_{i}-r s_{i}\right)$. (Note that we build $C$ on the values $S A^{-1}\left[n-S A^{\prime}[i]\right]$, not $S A^{-1}\left[n-S A^{\prime}[i]-(m-\ell+1)\right]$, because the latter depend on $\ell$ and all the suffixes in this range share their first $m+\ell$ symbols anyway, so the lexicographic comparison is the same.) Finally, point (4) on each $S A\left[d s_{i}, d s_{i}\right]$ is solved as for point (2), now on the constant-time $R M Q$ structure for the LCP array of $T$. The total time is then the optimal $O\left(m / \log _{\sigma} n+c\right)$.

Theorem 2. Let $T$ be a text of length $n$ over an alphabet of size $\sigma$. Then there is a data of size $O(n)$ that finds the $c$ contextual occurrences of $(P[1 . . m], \ell)$ in time $O\left(m / \log _{\sigma} n+c\right)$.

More generally, if we have an index that finds the suffix array range [rs..re] for $P$ in $T^{r e v}$, and can extract any cell of $S A, S A^{-1}$, and $S A^{\prime}$, we can use it for contextual reporting using our general solution. We need $O(n)$ extra bits for the various $R M Q$ data structures. Note we do not need to store $C$ explicitly because we can simulate it using $S A^{\prime}$ and $S A^{-1}$. Further, the arrays $L C P^{\prime}$ and $L C P$ are simulated with other $2 n+o(n)$ bits if we have access to $S A^{\prime}$ and $S A$ 24]. We then have the following result.

Theorem 3. Let $T$ be a text of length $n$ and an index on $T^{\text {rev }}$ using $\mathcal{S}$ bits of space that finds the suffix array range of $P[1 . . m]$ in time $t_{s}(m)$, and computes any cell of $S A, S A^{\prime}$, or $S A^{-1}$ in time $t_{S A}$, where $S A$ and $S A^{\prime}$ are the suffix arrays of $T$ and $T^{r e v}$, respectively. Then there is a data structure using $\mathcal{S}+O(n)$ bits of space that finds the $c$ contextual occurrences of $(P[1 . . m], \ell)$ in time $O\left(t_{s}(m)+c t_{S A}\right)$. 
Building on an index [3] that uses $n H_{k}\left(T^{r e v}\right)+o(n \log \sigma)+O(n)$ bits of space for any $k<\alpha \log _{\sigma} n$ and constant $0<\alpha<1$, where $H_{k}(S)<\log \sigma$ is the $k$ th order empirical entropy of string $S$ [19], we have $t_{s}(m)=O(m)$ and $t_{S A}=O(\log n)$. The index provides access to $S A^{\prime}$ and $\left(S A^{\prime}\right)^{-1}$ by storing their values at regular intervals of $T^{r e v}$, of length $s=\Theta(\log n)$ in our case, and marking the sampled positions of $S A^{\prime}$ in a bitvector. It provides a way to move in constant time from $i$ such that $S A^{\prime}[i]=j$ to $i^{\prime}=L F(i)$ such that $S A^{\prime}\left[i^{\prime}\right]=j-1$. Thus, if $S A^{\prime}[i]$ is not sampled, it can move $s^{\prime}<s$ times until finding a sampled cell $S A^{\prime}\left[L F^{s^{\prime}}(i)\right]=j^{\prime}$, and then $S A^{\prime}[i]=j^{\prime}+s^{\prime}$. The same $L F$ function is used $j^{\prime}-j<$ $s$ times, for $j^{\prime}=\lceil j / s\rceil \cdot s$, to find $\left(S A^{\prime}\right)^{-1}[j]$, by starting from the sampled value $\left(S A^{\prime}\right)^{-1}\left[j^{\prime}\right]$ and tracing it back to $\left(S A^{\prime}\right)^{-1}[j]=L F^{j^{\prime}-j}\left(\left(S A^{\prime}\right)^{-1}\left[j^{\prime}\right]\right)$. Enhancing it to computing values of $S A$ and $S A^{-1}$ (which correspond to $T$ ) requires to store their sampled values as well, because $T^{r e v}[j]=T[n-j]$. Finally, because $H_{k}(T)=H_{k}\left(T^{r e v}\right)$ [20, Sec. 11.3.2], we have the following result.

Theorem 4. Let $T$ be a text of length $n$ over an alphabet of size $\sigma$, with $k$ th order empirical entropy $H_{k}(T)$, for any $k<\alpha \log _{\sigma} n$ and constant $0<\alpha<1$. Then there is a data structure of $n H_{k}(T)+o(n \log \sigma)+O(n)$ bits that finds the c contextual occurrences of $(P[1 . . m], \ell)$ in time $O(m+c \log n)$.

We can speed up this index by using compact space, $O(n \log \sigma)$ bits (i.e., proportional to a plain representation of $T$ ). In this case, any cell of $S A$ or $S A^{-1}$ (and of $S A^{\prime}$ by building the structures on $T^{r e v}$ as well) can be computed in time $O\left(\log _{\sigma}^{\epsilon} n\right)$ for any constant $\epsilon>0$ [13. Further, this index finds the suffix array interval of $P$ in almost RAM-optimal time, $O\left(m / \log _{\sigma} n+\log _{\sigma}^{\epsilon} n\right)$.

Theorem 5. Let $T$ be a text of length $n$ over an alphabet of size $\sigma$. Then there is a data structure using $O(n \log \sigma)$ bits that finds the c contextual occurrences of $(P[1 . . m], \ell)$ in time $O\left(m / \log _{\sigma} n+(c+1) \log _{\sigma}^{\epsilon} n\right)$, for any constant $\epsilon>0$.

\section{Conclusions}

We have proposed a query that should be more meaningful than standard pattern locating in the case of highly repetitive text collections. Instead of simply locating all the positions of $T[1 . . n]$ where $P[1 . . m]$ appears, we give a context length $\ell$ and ask for the occurrences of all the $c$ distinct strings $X P Y$ in the text, for any $X, Y$ where $|X|=|Y|=\ell$. If $P$ occurs inside a highly repeated substring, many essentially identical occurrences will be reported one by one with the standard locating, whereas we will report only a single suffix array range comprising all the occurrences of the same context $X P Y$.

While the query can be solved in $O(n)$ space and RAM-optimal $O\left(m / \log _{\sigma} n+\right.$ $c$ ) time, we focus on using space proportional to the repetitiveness of $T$. We use one such measure, the number $r(S)$ of equal-letter runs of the BurrowsWheeler Transform of the string $S$. Within space $O(\bar{r} \log (n / \bar{r}))$, where $\bar{r}=$ $\max \left(r(T), r\left(T^{r e v}\right)\right)$, we solve the problem in time $O(m+$ occ log $n)$. We also show how to adapt our general strategy to any compressed text index. 
This is a first step towards studying queries that make more sense on highly repetitive text collections, possibly deviating from the classical ones used for regular collections. Some relevant remaining questions are: Can the obtained space/time tradeoffs be improved? Are there other relevant and challenging queries that are better suited to highly repetitive text collections?

\section{References}

1. Belazzougui, D., Cunial, F.: Representing the suffix tree with the CDAWG. In: Proc. 28th CPM. pp. 7:1-7:13 (2017)

2. Belazzougui, D., Cunial, F., Gagie, T., Prezza, N., Raffinot, M.: Composite repetition-aware data structures. In: Proc. 26th CPM. pp. 26-39 (2015)

3. Belazzougui, D., Navarro, G.: Alphabet-independent compressed text indexing. ACM Transactions on Algorithms 10(4), article 23 (2014)

4. Bille, P., Ettienne, M.B., Gørtz, I.L., Vildhøj, H.W.: Time-space trade-offs for Lempel-Ziv compressed indexing. Theoretical Computer Science 713, 66-77 (2018)

5. Blumer, A., Blumer, J., Haussler, D., McConnell, R.M., Ehrenfeucht, A.: Complete inverted files for efficient text retrieval and analysis. Journal of the ACM 34(3), 578-595 (1987)

6. Burrows, M., Wheeler, D.: A block sorting lossless data compression algorithm. Tech. Rep. 124, Digital Equipment Corporation (1994)

7. Christiansen, A.R., Ettienne, M.B., Kociumaka, T., Navarro, G., Prezza, N.: Optimal-time dictionary-compressed indexes. CoRR 1811.12779 (2019)

8. Claude, F., Navarro, G.: Improved grammar-based compressed indexes. In: Proc. 19th SPIRE. pp. 180-192 (2012)

9. Ferrada, H., Kempa, D., Puglisi, S.J.: Hybrid indexing revisited. In: Proc. 20th ALENEX. pp. 1-8 (2018)

10. Fischer, J., Heun, V.: Space-efficient preprocessing schemes for range minimum queries on static arrays. SIAM Journal on Computing 40(2), 465-492 (2011)

11. Gagie, T., Gawrychowski, P., Kärkkäinen, J., Nekrich, Y., Puglisi, S.J.: LZ77-based self-indexing with faster pattern matching. In: Proc. 11th LATIN. pp. 731-742 (2014)

12. Gagie, T., Navarro, G., Prezza, N.: Fully-functional suffix trees and optimal text searching in BWT-runs bounded space. Journal of the ACM 67(1), article 2 (2020)

13. Grossi, R., Vitter, J.S.: Compressed suffix arrays and suffix trees with applications to text indexing and string matching. SIAM Journal on Computing 35(2), 378-407 (2006)

14. Kempa, D., Kociumaka, T.: Resolution of the Burrows-Wheeler Transform conjecture. CoRR 1910.10631 (2019)

15. Kempa, D., Prezza, N.: At the roots of dictionary compression: String attractors. In: Proc. 50th STOC. pp. 827-840 (2018)

16. Kreft, S., Navarro, G.: On compressing and indexing repetitive sequences. Theoretical Computer Science 483, 115-133 (2013)

17. Mäkinen, V., Navarro, G., Sirén, J., Välimäki, N.: Storage and retrieval of highly repetitive sequence collections. Journal of Computational Biology 17(3), 281-308 (2010)

18. Manber, U., Myers, G.: Suffix arrays: A new method for on-line string searches. SIAM Journal on Computing 22(5), 935-948 (1993) 
19. Manzini, G.: An analysis of the Burrows-Wheeler transform. Journal of the ACM 48(3), 407-430 (2001)

20. Navarro, G.: Compact Data Structures - A practical approach. Cambridge University Press (2016)

21. Navarro, G.: Indexing highly repetitive string collections. CoRR abs/2004.02781 (2020)

22. Navarro, G., Nekrich, Y.: Time-optimal top- $k$ document retrieval. SIAM Journal on Computing 46(1), 89-113 (2017)

23. Navarro, G., Prezza, N.: Universal compressed text indexing. Theoretical Computer Science 762, 41-50 (2019)

24. Sadakane, K.: Compressed suffix trees with full functionality. Theory of Computing Systems 41(4), 589-607 (2007)

25. Takabatake, Y., Tabei, Y., Sakamoto, H.: Improved ESP-index: A practical selfindex for highly repetitive texts. In: Proc. 13th SEA. pp. 338-350 (2014)

26. Weiner, P.: Linear Pattern Matching Algorithms. In: Proc. 14th FOCS. pp. 1-11 (1973) 